\title{
CARACTERIZAÇÃO BIOQUÍMICA DE LINFONODOS NORMAIS, COMPROMETIDOS E LESÕES CERVICAIS POR ESPECTROSCOPIA RAMAN
}

\author{
Juliana dos Santos Corrêa ${ }^{1}$ \\ Lázaro Pinto Medeiros Neto ${ }^{2}$ \\ Laurita dos Santos ${ }^{3}$ \\ Luis Felipe das Chagas da Silva de Carvalho ${ }^{4}$ \\ Vinícius de Almeida Loddi ${ }^{5}$ \\ Claudio Alberto Tellez Soto ${ }^{6}$ \\ Airton Abrahão Martin ${ }^{7}$
}

Resumo: Os linfonodos fazem parte do conjunto de órgãos linfoides pertencentes ao sistema linfático, considerados os filtros da linfa por serem ricos em células de defesa. Uma das causas de seu comprometimento é a invasão de células neoplásicas. A biópsia com posterior exame histológico constitui o exame padrão ouro no diagnóstico. Porém, estudos questionam a eficácia desta técnica, devido a perda da arquitetura celular. Neste sentido, o trabalho teve como objetivo mostrar as diferenças entre linfonodos normais e comprometidos por células tumorais. Para isto, foi utilizado a espectroscopia Raman na caracterização bioquímica de linfonodos normais e comprometidos além de lesões cervicais. Os resultados mostraram uma taxa de sensibilidade de $71,4 \%$, especificidade de $80 \%$ e acurácia de $75 \%$ na discriminação dos tecidos. Portanto, a espectroscopia Raman se mostrou uma importante ferramenta no auxílio diagnóstico.

Palavras-chave: Espectroscopia Raman; Linfonodo; Linfoma; Sistema Linfático.

\footnotetext{
${ }^{1}$ Biomedicina/Laboratório de Espectroscopia Vibracional Biomédica/Universidade do Vale do Paraíba, Brasil. Email: julianasantocorrea@gmail.com.

2 Biomedicina/Laboratório de Espectroscopia Vibracional Biomédica/Universidade do Vale do Paraíba, Brasil. Email: Ipmn_777@yahoo.com.br.

${ }^{3}$ Biomedicina/Laboratório de Espectroscopia Vibracional Biomédica/Universidade do Vale do Paraíba, Brasil. Email: lauritas9@gmail.com.

${ }^{4}$ Biomedicina/Laboratório de Espectroscopia Vibracional Biomédica/Universidade do Vale do Paraíba, Brasil. Email: luisfelipecarvalho@hotmail.com.

${ }^{5}$ Biomedicina/Laboratório de Espectroscopia Vibracional Biomédica/Universidade do Vale do Paraíba, Brasil. Email: vloddi@hotmail.com.

${ }^{6}$ Biomedicina/Laboratório de Espectroscopia Vibracional Biomédica/Universidade do Vale do Paraíba, Brasil. Email: cayotellez@gmail.com.

${ }^{7}$ Biomedicina/Laboratório de Espectroscopia Vibracional Biomédica/Universidade do Vale do Paraíba, Brasil. Email: amartin@univap.br.
} 